\title{
Placement with Symmetry Constraints for Analog Layout Design Using TCG-S*
}

\author{
Jai-Ming Lin ${ }^{1}$, Guang-Ming Wu ${ }^{2}$, Yao-Wen Chang ${ }^{3}$, and Jen-Hui Chuang ${ }^{4}$ \\ ${ }^{1}$ Realtek Semiconductor Corp., Science-Based Industrial Park, Hsinchu, Taiwan \\ ${ }^{2}$ Department of Information Management, Nan-Hua University, Chiayi, Taiwan \\ ${ }^{3}$ Department of Electrical Engineering \& Graduate Institute of Electronics Engineering, National Taiwan University, Taipei 106, Taiwan \\ ${ }^{4}$ Department of Computer and Information Science, National Chiao Tung University, Hsinchu, Taiwan
}

\begin{abstract}
In order to handle device matching for analog circuits, some pairs of modules need to be placed symmetrically with respect to a common axis. In this paper, we deal with the module placement with symmetry constraints for analog design using the Transitive Closure Graph-Sequence (TCG-S) representation. Since the geometric relationships of modules are transparent to TCG-S and its induced operations, TCG-S has better flexibility than previous works in dealing with symmetry constraints. We first propose the necessary and sufficien conditions of TCG-S for symmetry modules. Then, we propose a polynomialtime packing algorithm for a TCG-S with symmetry constraints. Experimenta results show that the TCG-S based algorithm results in the best area utilization.
\end{abstract}

\section{Introduction}

In the design of analog circuits, it is often required that modules (devices) be placed symmetrically with respect to one or several common axes. If the parasitics in differential analog circuits do not match, it may lead to higher offset voltages and degraded power-supply rejection ratio [5]. Placing devices symmetrically can also reduce the circuit sensitivity to thermal gradients; failure to balance thermal couplings in a differential circuit may introduce unwanted oscillations [4]. Therefore, it is desirable to develop an efficient and effective approach to place symmetry modules for analog circuit designs.

The problem of placement with symmetry modules has been extensively studied in the literature $[2,3,4,5,9,12,14]$. Most of these works used the simulated annealing algorithm in combination with floorplan representations to handle symmetry constraints. We can classify these representations into two categories: (1) the absolute representation and (2) the topological representations.

The absolute representation was proposed by Jepsen and Gellat [7]. Fo this representation, each module is associated with an absolute coordinate on a gridless plane. We can operate on a module by changing its coordinate directly. The KOAN/ANAGRAM II [5], PUPPY-A [12], and LAYLA [9] systems al adopted the absolute representation to handle the placement of analog modules The main weakness of the absolute method lies in the fact that it may generate an infeasible placement with overlapped modules. Therefore, a post-processing step must be performed to eliminate this condition, implying a longer computation time and lower solution quality.

Unlike the absolute representation that operates on modules' absolute coordinates, the topological representations describes a placement by keeping the relative positions between modules. Therefore, it is often harder, but more flexible to model the symmetry constraints using the topological representations. For the topological representations, recently, several representations that can model non-slicing foorplans including sequence pairs [13], O-tree [6], and binary trees $[3,4]$ were used to handle the symmetry constraints. Murata et al. in [13] used two sequences of module names, namely sequence pairs, to represent the geometric relations of modules for general floorplan design. Balasa [2] then applied the sequence pairs to deal with the symmetry constraint Guo et al. in [6] proposed the O-tree representation for a left and bottom compacted placement. Pang et al. [14] used the O-tree representation to deal with the symmetry constraints. The feasibility of the O-tree solutions can only be detected after packing; therefore, they have to explore the whole solution space to find feasible solutions with the symmetry constraints, implying a longer running time. Balasa [3] transformed an O-tree representation into a binary tree representation for non-slicing floorplans. Unlike O-tree that does not restrict the exploration space, they propose a feasibility condition for the binary tree representation with symmetry constraints, and thus only feasible solutions are searched Chang et al. in [1] presented a $B^{*}$-tree representation for non-slicing floorplans Balasa et al. recently augmented the $\mathrm{B}^{*}$-tree, called the segment tree [4], to handle the symmetry constraints efficiently. Although the tree-based representations have relatively smaller solution space and a faster packing scheme than sequence pairs, they can only represent the compacted floorplan - a proper subset of the general floorplan, which implies that the optimal solution may be lost.

In this paper, we deal with the symmetry constraints using the Transitive Closure Graph-Sequence (TCG-S) representation. One major strength of TCG-

* Yao-Wen Chang's work was partially supported by the National Science Council of Taiwan under Grant No's. NSC 92-2215-E-002-018, NSC-93-2220E-002-001, and NSC 93-2752-E-002-008-PAE and by a grant from SprintSoft Inc.
$\mathrm{S}$ lies in the property that the geometric relationship of modules is transparent So TCG-S and its induced operations, implying that any violation of the symmetry constraints during perturbation can easily be detected and thus infeasible solutions can be discarded earlier. Therefore, TCG-S has better flexibility than previous works in dealing with symmetry constraints. We first develop the necessary and sufficient conditions of TCG-S for symmetry modules. Then, we propose an $O\left(m^{2}\right)$-time packing algorithm for a TCG-S with symmetry constraints, where $m$ is the number of modules. Experimental results show that the TCG-S based algorithm results in the best area utilization.

It should be noted that the packing times for the previous works with symmetry constraints are $O\left(\mathrm{~m}^{2}\right)$ for sequence pair [2], $O\left(\mathrm{~m}^{2}\right)$ for the O-tree [14], and $O(m \lg m)$ for the $\mathrm{B}^{*}$-tree (the segment tree) [4]. The $\mathrm{B}^{*}$-tree based method has lower packing time complexity, but, like the $\mathrm{O}$-tree, it is not $\mathrm{P}^{*}$ admissible [11] and can handle only compacted floorplan (which is a proper subset of the general floorplan).

The remainder of this paper is organized as follows. Section 2 formulates the placement problem with symmetry constraints. Section 3 reviews the TCG$S$ representation. Section 4 presents the feasibility conditions of TCG-S and a packing algorithm for placement with symmetry constraints. Section 5 introduces the perturbations for symmetry constraints. Experimental results are reported in Section 6, and concluding remarks are given in Section 7.

\section{Preliminaries}

Let $B=\left\{b_{1}, b_{2}, \ldots, b_{m}\right\}$ be a set of $m$ rectangular modules whose width, height, and area are denoted by $W_{i}, H_{i}$, and $A_{i}, 1<i<m$. Let $\left(X_{i}, Y_{i}\right)$ $\left(\left(X_{i}^{\prime}, Y_{i}^{\prime}\right)\right)$ denote the coordinate of the bottom-left (top-right) corner of module $b_{i}, 1 \leq i \leq m$, on a chip. Symmetry constraints can be formulated in terms of symmetry pairs and symmetry groups. A symmetry pair $\left(b_{i_{B}}, b_{i_{T}}\right)$ is a pair of modules with the same dimensions that have to be placed symmetrically with respect to an axis, where $b_{i_{B}}\left(b_{i_{T}}\right)$ denotes the symmetry module in the bottom (top) side. A symmetry group $\left\{\left(b_{1_{B}}, b_{1_{T}}\right),\left(b_{2_{B}}, b_{2_{T}}\right), \ldots,\left(b_{k_{B}}, b_{k_{T}}\right)\right\}$ consists of a set of symmetry pairs. For easier presentation, we consider the common axes horizontal of symmetry groups; however, similar method can be applied if the axes are in the $x$ axis. Let $Y_{i_{M}}$ denote the middle axis for a symmetry pair $\left(b_{i_{B}}, b_{i_{T}}\right)$ (i.e., $\left.2 Y_{i_{M}}=Y_{i_{B}}^{\prime}+Y_{i_{T}}\right)$. Given a symmetry group, the $x$ coordinates of the modules in each symmetry pair must be the same. Besides, they have to be placed symmetrically with respect to a common axis in the final placement, which implies the following equations:

$$
\begin{aligned}
X_{i_{B}}=X_{i_{T}} & \forall i=1, \ldots, k, \\
Y_{g} & =Y_{i_{M}} \quad \forall i=1, \ldots, k,
\end{aligned}
$$

where $Y_{g}$ is the coordinate of the common axis for the symmetry group. A placement $P$ is an assignment of $\left(X_{i}, Y_{i}\right), i=1, \ldots, m$, for each $b_{i}$ such that no two modules overlap and the symmetry constraints are satisfied as well. The goal of placement with symmetry modules is to optimize a predefined cost metric such as the resulting area (i.e., the minimum bounding rectangle of $P$ ) induced by a placement.

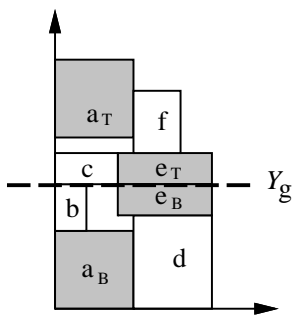

Figure 1: A feasible placement with a symmetry group $\left\{\left(b_{a_{B}}, b_{a_{T}}\right),\left(b_{e_{B}}, b_{e_{T}}\right)\right\}$.

Figure 1 shows a feasible placement with symmetry modules. There are eight modules $b_{a_{B}}, b_{a_{T}}, b_{b}, b_{c}, b_{d}, b_{e_{B}}, b_{e_{T}}$, and $b_{f}$ in the placement, where $\left(b_{a_{B}}, b_{a_{T}}\right)$ and $\left(b_{e_{B}}, b_{e_{T}}\right)$ are symmetry pairs in a symmetry group. 


\section{Review of TCG-S}

We first review the TCG-S representation presented in [11]. TCG-S describes the geometric relations among modules based on two graphs, namely a horizontal transitive closure graph $C_{h}$ and a vertical transitive closure graph $C_{v}$, and a packing sequence $\Gamma_{-}$. A node $n_{i}$ in $C_{h}\left(C_{v}\right)$ represents a module $b_{i}$ and an edge $\left(n_{i}, n_{j}\right)$ denotes that module $b_{i}$ is left to (below) module $b_{j}$. TCG-S has the following feasibility properties:

1. $C_{h}$ and $C_{v}$ are acyclic.

2. Each pair of nodes must be connected by exactly one edge either in $C_{h}$ or in $C_{v}$.

3. The transitive closure of $C_{h}\left(C_{v}\right)$ is equal to $C_{h}\left(C_{v}\right)$ itself. $^{1}$

4. The packing sequence $\Gamma_{-}$is the topological ordering of $C_{h}$ and $C_{v}$.

Figure 2(a) shows a placement with eight modules $a_{B}, a_{T}, b, c, d, e_{B}$, $e_{T}$, and $f$ whose widths and heights are $(5,5),(5,5),(2,3),(4,2),(5,6),(6,2)$ $(6,2)$, and $(3,4)$, respectively. Figure $2(\mathrm{~b})$ shows the TCG-S $=\left(C_{h}, C_{v}, \Gamma_{-}\right)$ corresponding to the placement of Figure 2(a). The value associated with a node in $C_{h}\left(C_{v}\right)$ gives the width (height) of the corresponding module, and the edge $\left(n_{i}, n_{j}\right)$ in $C_{h}\left(C_{v}\right)$ denotes the horizontal (vertical) relation of $b_{i}$ and $b_{j}$ Since there exists an edge $\left(n_{a_{B}}, n_{d}\right)$ in $C_{h}$, module $a_{B}$ is left to module $d$. Similarly, module $a_{B}$ is below module $b$ since there exists an edge $\left(n_{a_{B}}, n_{b}\right)$ in $C_{v}$. The packing sequence $\Gamma_{-}=\left\{a_{B}, b, d, e_{B}, c, e_{T}, a_{T}, f\right\}$ corresponds to the topological ordering of $C_{h}$ and $C_{v}$.

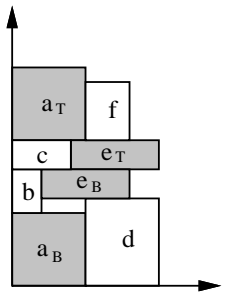

(a)

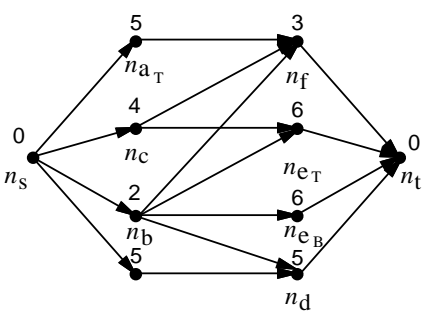

Augmented $C_{h}$

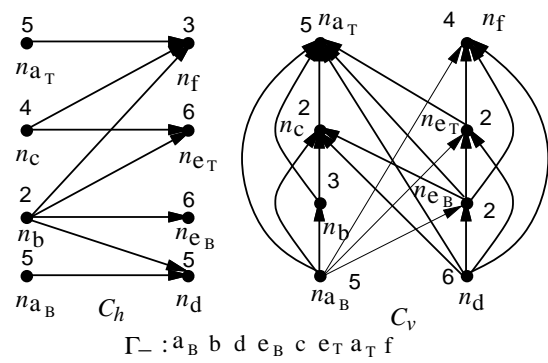

(b)

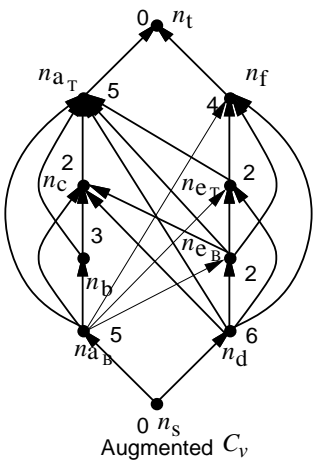

Figure 2: (a) A placement. (b) TCG-S. (c) Augmented $C_{h}$ and $C_{v}$.

Given a TCG-S, a placement can be obtained in $O\left(\mathrm{~m}^{2}\right)$ time by perform ing a well-known longest path algorithm [10] on the TCG-S, where $m$ is the number of modules. To facilitate the implementation of the longest path algorithm, the two closure graphs can be augmented as follows. For each closure graph, we introduce two special nodes, the source $n_{s}$ and the sink $n_{t}$, both with zero weights, and construct edges from $n_{s}$ to each node with in-degree equal to zero as well as from each node with out-degree equal to zero to $n_{t}$. (Note that the augmentation is performed only for packing.) Figure 2(c) shows the augmented $C_{h}$ and $C_{v}$ for the $C_{h}$ and $C_{v}$ shown in Figure 2(b). The longes path algorithm executes as follows: we process modules according to their topological ordering in the augmented $C_{h}\left(C_{v}\right)$. The $x(y)$ coordinate of dummy module $b_{s}$ is zero (i.e., $X_{s}=0\left(Y_{s}=0\right)$ ). For each module $b_{i}$ in the topological ordering, we serially relax it as follows: $X_{j}=\max \left\{X_{j}, X_{i}+W_{i}\right\}$ $\left(Y_{j}=\max \left\{Y_{j}, Y_{i}+H_{i}\right\}\right)$ if $n_{j} \in F_{\text {out }}\left(n_{i}\right)$. Let $L_{h}\left(n_{i}\right)\left(L_{v}\left(n_{i}\right)\right)$ denote the weight of the longest path from $n_{s}$ to $n_{i}$ in the augmented $C_{h}\left(C_{v}\right)$. The coordinate $\left(X_{i}, Y_{i}\right)$ of a module $b_{i}$ is given by $\left(L_{h}\left(n_{i}\right), L_{v}\left(n_{i}\right)\right)$. Since the respective width and height of the placement for the given TCG-S are $L_{h}\left(n_{t}\right)$ and $L_{v}\left(n_{t}\right)$, the area of the placement is given by $L_{h}\left(n_{t}\right) L_{v}\left(n_{t}\right)$. Since each module has a unique coordinate after packing, there exists a unique placement corresponding to any TCG-S.

\section{TCG-S for Symmetry Constraints}

In this section, we first introduce necessary and sufficient conditions of feasible TCG-S for the symmetry constraints.

${ }^{1}$ The transitive closure of a directed acyclic graph $G$ is defined as the graph $G^{\prime}=\left(V, E^{\prime}\right)$, where $E^{\prime}=\left\{\left(n_{i}, n_{j}\right)\right.$ : there is a path from node $n_{i}$ to node $n_{j}$ in $G$ \}.

\subsection{Feasible TCG-S}

In [11], we had shown that there always exists a unique feasible placement corresponding to a TCG-S for rectangular modules. However, for a TCG-S with symmetry modules, we must satisfy Equations (1) and (2) mentioned in Section 2 in the final placement. Therefore, we shall add two additional feasibility constraints for a TCG-S with symmetry constraints as follows:

5. Essence constraint: for modules $b_{i_{B}}$ and $b_{i_{T}}$ in each symmetry pair, the corresponding edge $\left(n_{i_{B}}, n_{i_{T}}\right)$ must be in $C_{v}$.

6. Homology constraint: for two arbitrary symmetry pairs $\left(b_{i_{B}}, b_{i_{T}}\right)$ and $\left(b_{j_{B}}, b_{j_{T}}\right)$ in a symmetry group, one of the following conditions is satisfied: (1) edges $\left(n_{i_{B}}, n_{j_{B}}\right)$ and $\left(n_{i_{T}}, n_{j_{T}}\right)$ are both in $C_{h},(2)$ edges $\left(n_{j_{B}}, n_{i_{B}}\right)$ and $\left(n_{j_{T}}, n_{i_{T}}\right)$ are both in $C_{h}$, (3) edges $\left(n_{i_{B}}, n_{j_{B}}\right)$ and $\left(n_{j_{T}}, n_{i_{T}}\right)$ are both in $C_{v}$, and (4) edges $\left(n_{j_{B}}, n_{i_{B}}\right)$ and $\left(n_{i_{T}}, n_{j_{T}}\right)$ are both in $C_{v}$

A TCG-S is called symmetric feasible iff Conditions 1-6 are satisfied. For the essence constraint, if $b_{i_{B}}$ and $b_{i_{T}}$ are the modules in a symmetry pair, $b_{i_{B}}$ must be directly below $b_{i_{T}}$ in order to have the same $x$ coordinates. There must exist an edge $\left(n_{i_{B}}, n_{i_{T}}\right)$ in $C_{v}$ according to the definition of TCG-S. In contrast, if edge $\left(n_{i_{B}}, n_{i_{T}}\right)$ (or $\left.\left(n_{i_{T}}, n_{i_{B}}\right)\right)$ is in $C_{h}$, the $x$ coordinates of $b_{i_{B}}$ and $b_{i_{T}}$ cannot be the same since $b_{i_{B}}\left(b_{i_{T}}\right)$ is in the left side of $b_{i_{T}}\left(b_{i_{B}}\right)$. For the homology constraint, if $b_{i_{B}}$ is left to $b_{j_{B}}, b_{i_{T}}$ must be left to $b_{j_{T}}$ such that the $x$ coordinates of $b_{i_{B}}$ and $b_{i_{T}}\left(b_{j_{B}}\right.$ and $\left.b_{j_{T}}\right)$ are the same. This implies that the edges $\left(n_{i_{B}}, n_{j_{B}}\right)$ and $\left(n_{i_{T}}, n_{j_{T}}\right)$ are in $C_{h}$. The $x$ coordinates of $b_{i_{B}}$ and $b_{i_{T}}\left(b_{j_{B}}\right.$ and $\left.b_{j_{T}}\right)$ are not the same if $b_{i_{B}}$ is left to $b_{j_{B}}$ but $b_{i_{T}}$ is right to $b_{j_{T}}$. Also, if $b_{i_{T}}$ is above $b_{j_{T}}, b_{j_{B}}$ must be above $b_{i_{B}}$ such that the middle axes of the two symmetry pairs can be between $b_{j_{B}}$ and $b_{j_{T}}$. This implies that edges $\left(n_{j_{T}}, n_{i_{T}}\right)$ and $\left(n_{i_{B}}, n_{j_{B}}\right)$ are in $C_{v}$. In contrast, the middle axis of $\left(b_{i_{B}}, b_{i_{T}}\right)$ is always above that of $\left(b_{j_{B}}, b_{j_{T}}\right)$ if $b_{i_{T}}$ is above $b_{j_{T}}$ and $b_{i_{B}}$ is above $b_{j_{B}}$

Figures 3(a) and (b) show a TCG-S with the symmetry group $\left\{\left(b_{a_{B}}, b_{a_{T}}\right)\right.$, $\left.\left(b_{e_{B}}, b_{e_{T}}\right)\right\}$ and the corresponding placement. The TCG-S shown in Figure 3(a) is feasible for the symmetry modules since the essence constraint (i.e., edges $\left(n_{a_{B}}, n_{a_{T}}\right)$ and $\left(n_{e_{B}}, n_{e_{T}}\right)$ are in $\left.C_{v}\right)$ and the homology constraint (i.e., edges $\left(n_{a_{B}}, n_{e_{B}}\right)$ and $\left(n_{e_{T}}, n_{a_{T}}\right)$ are in $\left.C_{v}\right)$ are satisfied. (Note that the modules $b_{e_{B}}$ and $b_{e_{T}}$ shown in Figure 3(b) are not symmetric. We will discuss how to make them symmetric later.) For the TCG-S shown in Figures 3(c), Condition (1) of the homology constraint is violated since edge $\left(n_{a_{T}}, n_{e_{T}}\right)$ is in $C_{h}$ but $\left(n_{a_{B}}, n_{e_{B}}\right)$ is not. This makes $b_{a_{T}}$ in the left side of $b_{e_{T}}$ and $b_{a_{B}}$ in the right side of $b_{e_{B}}$ in the resulting placement shown in Figures 3(d), and thus $b_{a_{B}}$ and $b_{a_{T}}\left(b_{e_{B}}\right.$ and $\left.b_{e_{T}}\right)$ cannot have the same $x$ coordinate. Similarly, Condition (3) of the homology constraint is violated for the TCG-S shown in Figure 3(e) because edge $\left(n_{a_{T}}, n_{e_{T}}\right)$ is in $C_{v}$ while edge $\left(n_{e_{B}}, n_{a_{B}}\right)$ is not. Since the middle axis of $\left(b_{e_{B}}, b_{e_{T}}\right)$ is always higher than that of $\left(b_{a_{B}}, b_{a_{T}}\right)$ in the resulting placement shown in Figure 3(f), the two symmetry pairs cannot have the same middle axis.

\subsection{Packing}

As mentioned in Section 3, the packing of regular modules can be obtained by applying the longest path algorithm on the augmented transitive closure graph. To guarantee a feasible placement with symmetry constraints, however, we need to modify the packing algorithm. Figure 4 illustrates the difference between the packings with and without symmetry modules. Figures 4(a) and (b) give a TCG-S with a symmetry group $\left\{\left(b_{a_{B}}, b_{a_{T}}\right),\left(b_{e_{B}}, b_{e_{T}}\right)\right\}$ and its corresponding placement. The placement is not correct for the symmetry modules because $X_{e_{B}} \neq X_{e_{T}}$ and $Y_{e_{M}} \neq Y_{a_{M}}$. The correct one is shown in Figure 4(d).

For each symmetry pair $\left(b_{i_{B}}, b_{i_{T}}\right)$, the $x$ coordinates of $b_{i_{B}}$ and $b_{i_{T}}$ must be the same. To make $X_{i_{B}} \stackrel{B}{=} X_{i_{T}}$, in addition to the longest path from the source to $b_{i_{B}}\left(b_{i_{T}}\right)$ in the augmented $C_{h}$, we also have to consider the $x$ coordinate of $b_{i_{T}}\left(b_{i_{B}}\right)$. For the placement shown in Figure 4(b), the $x$ coordinates of $b_{e_{B}}$ and $b_{e_{T}}$ are different since $b_{e_{T}}$ are in the right side of both $b_{b}$ and $b_{c}$ while $b_{e_{B}}$ is in the right side of only $b_{b}$ according to the TCG-S of Figure 4(a). If we make $b_{e_{B}}$ and $b_{e_{T}}$ both in the right side of $b_{b}$ and $b_{c}$, their $x$ coordinates will be the same (see Figure 4(c) for the resulting placement). Let the fan-in (fan-out) of a node $n_{i}$, denoted by $F_{i n}\left(n_{i}\right)\left(F_{\text {out }}\left(n_{i}\right)\right)$, be the nodes $n_{j}$ 's with edges $\left(n_{j}, n_{i}\right)\left(\left(n_{i}, n_{j}\right)\right)$. For the modules in each symmetry pair $\left(b_{i_{B}}, b_{i_{T}}\right)$, we have to add dummy edge $\left(n_{j}, n_{i_{B}}\right)\left(\left(n_{j}, n_{i_{T}}\right)\right)$ to $C_{h}$ if $n_{j} \in F_{i n}\left(n_{i_{T}}\right)$ and $n_{j} \notin F_{i n}\left(n_{i_{B}}\right)\left(n_{j} \in F_{i n}\left(n_{i_{B}}\right)\right.$ and $\left.n_{j} \notin F_{i n}\left(n_{i_{T}}\right)\right)$ before applying the longest path algorithm on the graph. After the $x$ coordinate of each module is determined, those newly added edges are removed from $C_{h}$ to guarantee the correctness of TCG-S during perturbation.

For each symmetry pair $\left(b_{i_{B}}, b_{i_{T}}\right)$ in a symmetry group, the distance between $b_{i_{B}}$ and $Y_{g}$ must equal to that between $b_{i_{T}}$ and $Y_{g}$, where $Y_{g}$ is the common axis of the group. Let $\Delta\left(i_{T}\right)\left(\Delta\left(i_{B}\right)\right)$ denote the distance between the bottom (top) boundary of module $b_{i_{T}}\left(b_{i_{B}}\right)$ and $Y_{g}$ (i.e $\Delta\left(i_{T}\right)=Y_{i_{T}}-Y_{g}$ $\left.\left(\Delta\left(i_{B}\right)=Y_{g}-Y_{i_{B}}^{\prime}\right)\right)$. For the placement shown in Figure 4(c), two symmetry pairs $\left(b_{a_{B}}, b_{a_{T}}\right)$ and $\left(b_{e_{B}}, b_{e_{T}}\right)$ are not placed symmetrically with respect to the same axis since the original packing algorithm relaxes modules according to the sequence defined in $\Gamma_{-}$- without considering $\Delta\left(a_{B}\right)$ and $\Delta\left(a_{T}\right)$ $\left(\Delta\left(e_{B}\right)\right.$ and $\left.\Delta\left(e_{T}\right)\right)$ as their values. (See the last photography of Section 3 for the relax process.) If we first relax the symmetry pair $\left(b_{e_{B}}, b_{e_{T}}\right)$, and then the other $\left(b_{a_{B}}, b_{a_{T}}\right)$, we can make $\Delta\left(e_{B}\right)\left(\Delta\left(a_{B}\right)\right)$ equal to $\Delta\left(e_{T}\right)$ $\left(\Delta\left(a_{T}\right)\right)$ by choosing the larger one of the two values as their values. We first assume that the common axis of a symmetry group is the highest middle 

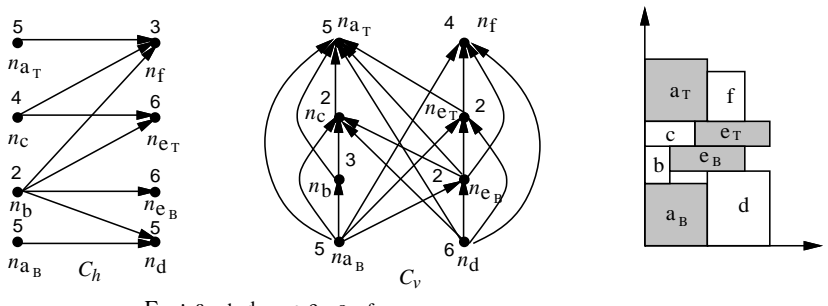

(a)
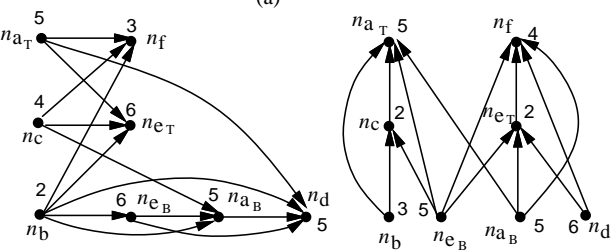

$C_{v}$

$\Gamma_{-}: \mathrm{be}_{\mathrm{B}} \mathrm{c} \mathrm{a}_{\mathrm{B}} \mathrm{a}_{\mathrm{T}} \mathrm{d} \mathrm{e}_{\mathrm{T}} \mathrm{f}$

(c)

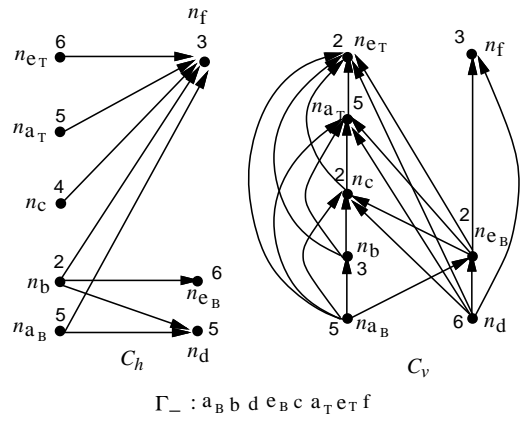

(e)
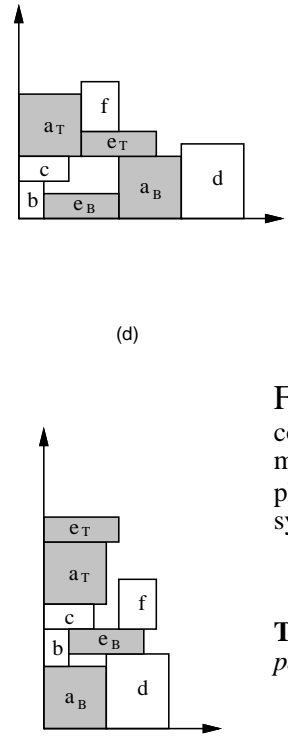

(f)
Figure 3: (a) A symmetric feasible TCG-S, (b) the placement corresponding to the TCG-S in (a), (c) a symmetric infeasible TCG-S (violation of Condition (1) of the homology constraint), (d) the placement corresponding to the TCG$\mathrm{S}$ in (c), (e) a symmetric infeasible TCG-S (violation of Condition (3) of the homology constraint), (f) the placement corresponding to the TCG-S in (e).

axis $Y_{j_{M}}$ for all symmetry pairs in the group. Therefore, for each symmetry pair $\left(b_{i_{B}}, b_{i_{T}}\right)$ in a symmetry group, the initial value for the distance between bottom (top) symmetry module and the common axis is $Y_{j_{M}}-Y_{i_{B}}^{\prime}$ (i.e., $\left.\Delta\left(i_{B}\right)=\Delta\left(i_{T}\right)=Y_{j_{M}}-Y_{i_{B}}^{\prime}\right)$. Also, the distance between other module $b_{w}$ and the common axis is $Y_{w}-Y_{j_{M}}\left(Y_{j_{M}}-Y_{w}^{\prime}\right)$ if $b_{w}$ is above (below) the common axis. Different from the original longest path algorithm that relaxes modules according to the sequence defined in $\Gamma_{-}$, we first relax modules $b_{l_{B}}$ and $b_{l_{T}}$ if $b_{l_{B}}$ is the last bottom symmetry module in $\Gamma_{-}$since there cannot exist any symmetry modules between $b_{l_{B}}$ and $b_{l_{T}}$. Note that a module is deleted from $\Gamma_{-}$if it is relaxed in this algorithm. We then relax the modules from $b_{l_{B}}$ to $b_{k_{B}}$ and from $b_{k_{B}}$ to $b_{k_{T}}$ if $b_{k_{B}}$ is the nearest bottom symmetry module in the left side of $b_{l_{B}}$ in $\Gamma_{-}$. The process is repeated until all modules in $\Gamma_{-}$are traversed. A module is said to be above-marked (below-marked) if it is a fan-out (fan-in) module of a relaxed module. During traversing modules from $b_{l_{B}}$ to $b_{k_{B}}$, those below-marked modules $b_{u}$ 's are relaxed for those modules belonging to fan-in's of $b_{u}$ 's. Then, we start to relax the above-marked modules $b_{v}$ 's for those modules belonging to fan-out's of $b_{v}$ 's until the top symmetry module $b_{k_{T}}$ is met. (Note that we only relax the marked modules since only those modules belonging to fan-in's (fan-out's) of symmetry modules are changed during our algorithm.) Then, we can make $\Delta\left(k_{B}\right)$ equal to $\Delta\left(k_{T}\right)$ by assigning $\Delta\left(k_{B}\right)=\Delta\left(k_{T}\right)=\max \left\{\Delta\left(k_{B}\right), \Delta\left(k_{T}\right)\right\}$. After $\Delta\left(k_{B}\right)$ and $\Delta\left(k_{T}\right)$ are obtained, $b_{k_{B}}$ and $b_{k_{T}}$ are relaxed and the same process repeats until the last module in $\Gamma_{-}$is met. After these processes, $\Delta\left(i_{B}\right)=\Delta\left(i_{T}\right)$ for each symmetry pair $\left(b_{i_{B}}, b_{i_{T}}\right)$ in the group. If the $y$ coordinate of the common axis is zero, the coordinates for those below-marked (above-marked) or bottom (top) symmetry modules $b_{p}$ 's $\left(b_{q}\right.$ 's) are $-\Delta(p)(\Delta(q))$. To make the coordinates of all modules larger than zero, we have to pull all modules upward by $\Delta(s)$ distance, and thus $Y_{p}^{\prime}=-\Delta(p)+\Delta(s)\left(Y_{q}=\Delta(q)+\Delta(s)\right)$, where $\Delta(s)$ is distance between the dummy module $b_{s}$ and the common axis. Finally, for those unprocessed modules $b_{x}$ 's, their new coordinates $Y_{x}=\max \left\{Y_{r}^{\prime}\right\}$ if $n_{r} \in F_{i n}\left(n_{x}\right)$ in $C_{v}$.

Theorem 1 Given a symmetric feasible TCG-S, the packing scheme gives a feasible placement of the minimum area in $O\left(m^{2}\right)$ time, where $m$ is the number of modules.

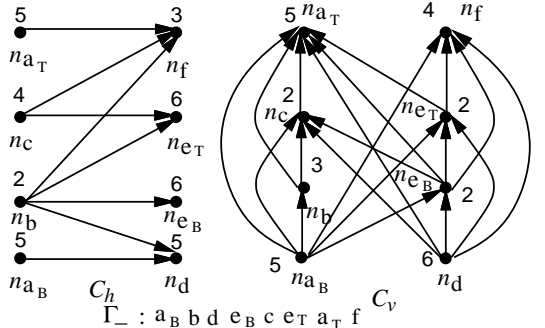

(a)

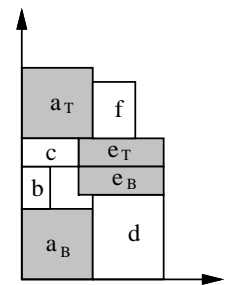

(c)

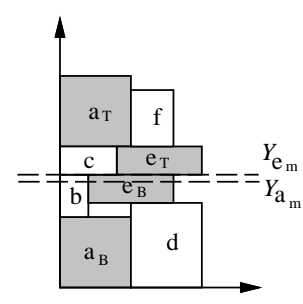

(b)

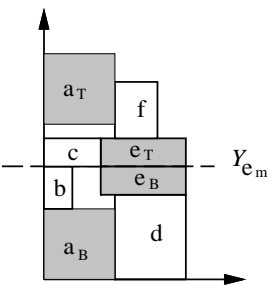

(d)
Figure 4: (a) and (b) A TCG-S and its corresponding placement without considering the symmetry pair $\left\{\left(b_{e_{B}}, b_{e_{T}}\right)\right\}$. (c) The resulting placement after modules $b_{e_{B}}$ and $b_{e_{T}}$ are aligned at the same $x$ coordinate. (d) The resulting placement after two symmetry pairs $\left(b_{a_{B}}, b_{a_{T}}\right)$ and $\left(b_{e_{B}}, b_{e_{T}}\right)$ are placed symmetrically with respect to the same axis $Y_{e_{m}}$.

Theorem 2 There exists a correspondence between a minimum area rectangle packing with symmetry constraints and a symmetric feasible TCG-S.

Note that the packing times for the previous works with symmetry constraints are $O\left(\mathrm{~m}^{2}\right)$ for sequence pair [2], $O\left(\mathrm{~m}^{2}\right)$ for the O-tree [14], and $O(m \lg m)$ for the $\mathrm{B}^{*}$-tree (the segment tree) [4]. The $\mathrm{B}^{*}$-tree based method has lower packing time complexity, but, like the $\mathrm{O}$-tree, it is not $\mathrm{P}^{*}$ admissible [11] and can handle only compacted floorplan (which is a proper subset of the general floorplan).

\section{Algorithm}

Our algorithm is based on simulated annealing [8]. Given an initial solution represented by a TCG-S, we perturb the TCG-S to obtain a new TCG-S. The perturbation continues to search for a "good" configuration until a predefined termination condition is satisfied. To ensure the correctness of a packing with symmetry modules, the new TCG-S must satisfy the TCG-S feasibility conditions described in Section 3 and the essence and the homology constraints presented in Section 4.1. The following five operations are introduced in [11] to perturb a TCG-S:

- Rotation: Rotate a rectangular module.

- Swap: Swap two nodes associated with two rectangular modules in both $C_{h}$ and $C_{v}$.

- Reverse: Reverse a reduction edge in $C_{h}$ or $C_{v}$.

- Move: Move a reduction edge from one transitive closure graph $\left(C_{h}\right.$ or $C_{v}$ ) to the other.

- Transpositional Move: Move a reduction edge from one transitive closure graph $\left(C_{h}\right.$ or $\left.C_{v}\right)$ to the other, and then transpose the two nodes associated with the edge.

We introduce the five operations that can perturb an arbitrary edge, and we show how to maintain the feasibility of symmetric feasible TCG-S in each perturbation as follows

\subsection{Rotation}

We cannot rotate node $n_{i}$ if $b_{i}$ denotes a symmetry module except the other symmetry module $b_{j}$ is rotated.

\subsection{Swap}

We cannot swap two nodes $n_{i}$ and $n_{j}$ if $b_{i}$ or $b_{j}$ denotes a symmetry module except the following two conditions:

- $n_{i}$ and $n_{j}$ denote modules in a symmetry pair.

- $n_{i}$ and $n_{j}$ denote two bottom (top) symmetry modules. Then, we have to swap the corresponding top (bottom) symmetry modules.

For the first condition, $b_{i}\left(b_{j}\right)$ is considered as top (bottom) symmetry module if it is a bottom (top) symmetry module originally.

\subsection{Reverse}

If we reverse a reduction edge $\left(n_{i}, n_{j}\right)$ (there does not exist another path from $n_{i}$ to $n_{j}$, except the edge $\left(n_{i}, n_{j}\right)$ itself) in a transitive closure graph $G$, the feasibility of TCG-S is violated under the following two conditions:

- $G=C_{h}$ : one fan-in of $n_{j}$ or $n_{j}$ denotes a bottom (top) symmetry module $b_{p_{B}}\left(b_{p_{T}}\right)$, and one fan-out of $n_{i}$ or $n_{i}$ denotes the other top (bottom) symmetry module $b_{p_{T}}\left(b_{p_{B}}\right)$. 


\begin{tabular}{|c|c|c|c|c|c|c|c|c|c|}
\hline \hline & \multirow{2}{*}{$\begin{array}{c}\text { \# of } \\
\text { Circuit }\end{array}$} & mod. & symmetry & Total & \multicolumn{2}{|c|}{ Sequence pairs } & \multicolumn{2}{c|}{ Segment tree } & \multicolumn{2}{c|}{ TCG-S } \\
\cline { 5 - 10 } & modules & area & Area & Time $(\mathrm{sec})$ & Area & Time (sec) & Area (\%dead space) & Time (sec) \\
\hline apte & 9 & 8 & 46.56 & 48.12 & 25 & 47.52 & 11 & $47.52(2.1 \%)$ & 3 \\
hp & 11 & 8 & 8.830 & 9.84 & 138 & 9.714 & 62 & $9.714(10.0 \%)$ & 50 \\
ami33 & 33 & 6 & 1.156 & 1.24 & 684 & 1.23 & 307 & $1.21(4.7 \%)$ & 423 \\
ami49 & 49 & 4 & 35.45 & 37.82 & 2038 & 37.31 & 983 & $37.04(4.5 \%)$ & 1247 \\
\hline \hline
\end{tabular}

Table 1: Experimental results for the benchmark circuits.

- $G=C_{v}$ : one fan-in of $n_{j}$ or $n_{j}$ denotes a top symmetry module $b_{p_{T}}$ and one fan-out of $n_{i}$ or $n_{i}$ denotes another bottom symmetry module $b_{q_{B}}$

If we reverse a reduction edge $\left(n_{i_{B}}, n_{j_{B}}\right)$ (or $\left(n_{i_{T}}, n_{j_{T}}\right)$ ) in $C_{h}$, the edge $\left(n_{i_{T}}, n_{j_{T}}\right)\left(\left(n_{i_{B}}, n_{j_{B}}\right)\right)$ must also be reversed to maintain the homology constraint. Besides, if a new edge $\left(n_{l_{B}}, n_{k_{B}}\right)$ (or $\left(n_{l_{T}}, n_{k_{T}}\right)$ ) is added to a $C_{h}$ during the operation, we must also move the edge $\left(n_{l_{T}}, n_{k_{T}}\right)$ $\left(\left(n_{l_{B}}, n_{k_{B}}\right)\right)$ from $C_{v}$ to $C_{h}$ if there exists an edge $\left(n_{l_{T}}, n_{k_{T}}\right)\left(\left(n_{l_{B}}, n_{k_{B}}\right)\right)$ is in $C_{v}$; otherwise, we should transpositionally move edge $\left(n_{k_{T}}, n_{l_{T}}\right)$ $\left(\left(n_{k_{B}}, n_{l_{B}}\right)\right)$ from $C_{v}$ to $C_{h}$ if the edge is in $C_{v}$. Similarly, if we reverse a reduction edge $\left(n_{i_{B}}, n_{j_{B}}\right)$ (or $\left.\left(n_{i_{T}}, n_{j_{T}}\right)\right)$ in $C_{V}$, the edge $\left(n_{j_{T}}, n_{i_{T}}\right)$ $\left(\left(n_{j_{B}}, n_{i_{B}}\right)\right)$ must also be reversed. Besides, if a new edge $\left(n_{l_{B}}, n_{k_{B}}\right)$ $\left(\left(n_{l_{T}}, n_{k_{T}}\right)\right)$ is added to a $C_{v}$ during this operation, we must also move the edge $\left(n_{k_{T}}, n_{l_{T}}\right)\left(\left(n_{k_{B}}, n_{l_{B}}\right)\right)$ to $C_{v}$ if there exists an edge $\left(n_{k_{T}}, n_{l_{T}}\right)$ $\left(\left(n_{k_{B}}, n_{l_{B}}\right)\right)$ in $C_{h}$; otherwise, we should transpositionally move an edge $\left(n_{l_{T}}, n_{k_{T}}\right)\left(\left(n_{l_{B}}, n_{k_{B}}\right)\right)$ from $C_{h}$ to $C_{v}$ if the edge is in $C_{h}$

\subsection{Move}

If we move a reduction edge $\left(n_{i}, n_{j}\right)$ from a transitive closure graph $G$ to the other $G^{\prime}$, the feasibility of TCG-S with symmetry modules is violated in the following conditions:

- $G^{\prime}=C_{h}$ : one fan-in of $n_{i}$ or $n_{i}$ denotes a bottom (top) symmetry module $b_{p_{B}}\left(b_{p_{T}}\right)$, and one fan-out of $n_{j}$ or $n_{j}$ denotes the other top (bottom) symmetry module $b_{p_{T}}\left(b_{p_{B}}\right)$.

- $G=C_{v}$ : one fan-in of $n_{i}$ or $n_{i}$ denotes a top symmetry module $b_{p_{T}}$, and one fan-out of $n_{j}$ or $n_{j}$ denotes another bottom symmetry module $b_{q_{B}}$.

It takes $O(m)$ time to detect the violation by checking the modules $b_{q}$ 's before $b_{i}$ and after $b_{j}$ according the sequence defined in $\Gamma_{-}$, where $m$ is number of modules.

If we move edge a reduction edge $\left(n_{i_{B}}, n_{j_{B}}\right)$ (or $\left(n_{i_{T}}, n_{j_{T}}\right)$ ) from $C_{v}$ to $C_{h}$, the edge $\left(n_{j_{T}}, n_{i_{T}}\right)\left(\left(n_{j_{B}}, n_{i_{B}}\right)\right)$ in $C_{v}$ must be transpositionally moved to $C_{h}$ to maintain the homology constraint. Besides, if a new edge $\left(n_{l_{B}}, n_{k_{B}}\right)$ (or $\left(n_{l_{T}}, n_{k_{T}}\right)$ ) is added to a $C_{h}$ during the operation, we must move the edge $\left(n_{l_{T}}, n_{k_{T}}\right)\left(\left(n_{l_{B}}, n_{k_{B}}\right)\right)$ to $C_{h}$ if there exists an edge $\left(n_{l_{T}}, n_{k_{T}}\right)\left(\left(n_{l_{B}}, n_{k_{B}}\right)\right)$ in $C_{v}$; otherwise, we should transpositionally move edge $\left(n_{k_{T}}, n_{l_{T}}\right)\left(\left(n_{k_{B}}, n_{l_{B}}\right)\right)$ from $C_{v}$ to $C_{h}$ if the edge is in $C_{v}$. Similarly, if we move a reduction edge $\left(n_{i_{B}}, n_{j_{B}}\right)$ (or $\left(n_{i_{T}}, n_{j_{T}}\right)$ ) from $C_{h}$ to $C_{v}$, the edge $\left(n_{i_{T}}, n_{j_{T}}\right)\left(\left(n_{i_{B}}, n_{j_{B}}\right)\right)$ in $C_{h}$ must be transpositionally moved to $C_{v}$ Besides, if a new edge $\left(n_{l_{B}}, n_{k_{B}}\right)\left(\left(n_{l_{T}}, n_{k_{T}}\right)\right)$ is added to a $C_{v}$ during this operation, we must move the edge $\left(n_{k_{T}}, n_{l_{T}}\right)\left(\left(n_{k_{B}}, n_{l_{B}}\right)\right)$ to $C_{v}$ if there exists an edge $\left(n_{k_{T}}, n_{l_{T}}\right)\left(\left(n_{k_{B}}, n_{l_{B}}\right)\right)$ in $C_{h}$; otherwise, we should transpositionally move edge $\left(n_{l_{T}}, n_{k_{T}}\right)\left(\left(n_{l_{B}}, n_{k_{B}}\right)\right)$ from $C_{h}$ to $C_{v}$ if the edge is in $C_{h}$.

\subsection{Transpositional Move}

If we transpositionally move a reduction edge $\left(n_{i}, n_{j}\right)$ from a transitive closure graph $G$ to the other $G^{\prime}$, the feasibility of TCG-S with symmetry modules is violated in the following conditions:

- $G^{\prime}=C_{h}$ : one fan-in of $n_{j}$ or $n_{j}$ denotes a bottom (top) symmetry module $b_{p_{B}}\left(b_{p_{T}}\right)$, and one fan-out of $n_{i}$ or $n_{i}$ denotes the other top (bottom) symmetry module $b_{p_{T}}\left(b_{p_{B}}\right)$.

- $G^{\prime}=C_{v}$ : one fan-in of $n_{j}$ or $n_{j}$ denotes a top symmetry module $b_{p_{T}}$, and one fan-out of $n_{i}$ or $n_{i}$ denotes another bottom symmetry module $b_{q_{B}}$.

If we transpositionally move a reduction edge $\left(n_{i_{B}}, n_{j_{B}}\right)\left(\right.$ or $\left(n_{i_{T}}, n_{j_{T}}\right)$ ) from $C_{v}$ to $C_{h}$, the edge $\left(n_{j_{T}}, n_{i_{T}}\right)\left(\left(n_{j_{B}}, n_{i_{B}}\right)\right)$ in $C_{v}$ must be also moved to $C_{h}$. Besides, if a new edge $\left(n_{l_{B}}, n_{k_{B}}\right)$ (or $\left(n_{l_{T}}, n_{k_{T}}\right)$ ) is added to a $C_{h}$ during the operation, we must move the edge $\left(n_{l_{T}}, n_{k_{T}}\right)\left(\left(n_{l_{B}}, n_{k_{B}}\right)\right)$ to $C_{h}$ if there exists an edge is $\left(n_{l_{T}}, n_{k_{T}}\right)\left(\left(n_{l_{B}}, n_{k_{B}}\right)\right)$ in $C_{v}$; otherwise, we should transpositionally move edge $\left(n_{k_{T}}, n_{l_{T}}\right)\left(\left(n_{k_{B}}, n_{l_{B}}\right)\right)$ to $C_{h}$ if the edge is in $C_{v}$. Similarly, if we transpositionally move a reduction edge $\left(n_{i_{B}}, n_{j_{B}}\right)$ (or $\left.\left(n_{i_{T}}, n_{j_{T}}\right)\right)$ from $C_{h}$ to $C_{v}$, the edge $\left(n_{i_{T}}, n_{j_{T}}\right)\left(\left(n_{i_{B}}, n_{j_{B}}\right)\right)$ in $C_{h}$ is also moved to $C_{v}$. Besides, if a new edge $\left(n_{l_{B}}, n_{k_{B}}\right)\left(\left(n_{l_{T}}, n_{k_{T}}\right)\right)$ is added to a $C_{v}$ during this operation, we must move the edge $\left(n_{k_{T}}, n_{l_{T}}\right)\left(\left(n_{k_{B}}, n_{l_{B}}\right)\right)$ to $C_{v}$ if there exists an edge is $\left(n_{k_{T}}, n_{l_{T}}\right)\left(\left(n_{k_{B}}, n_{l_{B}}\right)\right)$ in $C_{h}$; otherwise, we should transpositionally move an edge $\left(n_{l_{T}}, n_{k_{T}}\right)\left(\left(n_{l_{B}}, n_{k_{B}}\right)\right)$ to $C_{v}$ if the edge is in $C_{v}$.

\section{Experimental Results}

Based on the simulated annealing method [8], we implemented the placement algorithm using TCG-S in the $\mathrm{C}++$ programming language on a $433 \mathrm{MHz}$ SUN Sparc Ultra-60 workstation with 1 GB memory. The benchmarks (apte, hp, ami33, and ami49) in Table 1, we impose the symmetry constraints to the modules with the same dimensions in a set of commonly used MCNC benchmarks. As shown in Table 1, Columns 2 lists the number of modules of four benchmarks. Columns 3 lists the number of modules in symmetric group.

The total area of modules in each circuit are shown in Column 4. Columns 5,7 , and 9 list the respective resulting areas obtained by the sequence pairs (SP) method proposed in [2], the segment-tree method presented in [4], and our program, based on the same simulated annealing engine; Columns 6, 8, and 10 list their respective running times. As shown in the table, our method resulted in much more effective area utilization than the SP-based and the segment tree-based methods. Further, our method is also much more efficient than the SP-based method and comparable to the segment tree-based method. Figure 5 shows the resulting placement for ami33 (the shaded modules denote symmetry modules). The experimental results show that our TCG-based algorithm consistently obtained good results.

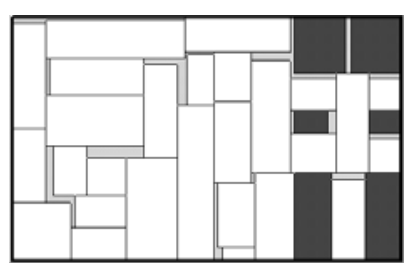

Figure 5: The resulting placement of ami33 with three symmetry pairs $\left(\right.$ area $\left.=1.219 \mathrm{~mm}^{2}\right)$.

\section{Concluding Remarks}

We have presented a TCG-S based algorithm to deal with the placement with symmetry constraints. TCG-S is the first general graph representation with the feasibility guarantee for each perturbation. We have derived necessary and sufficient conditions of TCG-S for symmetry modules, and proposed a packing algorithm for TCG-S. Experimental results have shown that our method is very efficient and effective.

\section{References}

1] Y.-C. Chang, et al, "B*-trees: A New Representation for Non-Slicing Floorplans," Proc. DAC, pp. 458-463, 2000.

[2] F. Balasa and K. Lampaert, "Symmetry Within the Sequence-Pair Representation in the Context of Placement for Analog Design," IEEE TCAD, vol. 19, no. 7, 721-731, July 2000

[3] F. Balasa, "Modeling Non-Slicing Floorplans with Binary Trees," ICCAD, 2000.

[4] F. Balasa, et al, "Efficient solution space exploration based on segment trees in analog placement for analog placement with sysmetry constraints," ICCAD, pp. 497$502,2002$.

[5] J. Cohn, et al, "KOAN/ANAGRAMII: New Tools for Device-Level Analog Layout," IEEE J. Solid-State Circuits, vol 26., PP. 330-342, Mar. 1991.

[6] P.-N. Guo, et al, "An O-Tree Representation of Non-Slicing Floorplan and Its Applications,"Proc. DAC, pp. 268-273, 1999.

[7] D. W. Jepsen and C. D. Gellat Jr., "Macro Placement by Monte Carlo Annealing," Proc. ICCD, pp. 495-485, Nov. 1983.

[8] S. Kirkpatrick, et al, "Optimization by Simulated Annealing," Science, vol. 220, no. 4598, May 13, 1983, pp.671-680.

[9] K. Lampaert, et al, "A Performance-Driven Placement Tool for Analog Integrated Circuits," IEEE J. Solid-State Circuits, vol. 30, pp. 773-780, July 1995

[10] E. Lawler, Combinatorial Optimization: Networks and Matroids, Holt, Rinehart, and Winston, 1976

[11] J.-M. Lin and Y.-W. Chang, "TCG-S: Orthogonal Coupling of P*-admissible Representations for General Floorplans," IEEE Trans. Computer-Aided Design, Vol. 24, No. 6, June 2004

[12] E. Malavasi, et al, "Automation of IC Layout with Analog Constraints," IEEE TCAD, vol. 15, pp. 923-942, Aug. 1996.

[13] H. Murata, et al, "Rectangle-Packing Based Module Placement," Proc. ICCAD, pp. 472-479, 1995

[14] Y. Pang, et al, "Block Placement with Symmetry Constraints based on thee O-tree Non-Slicing Representation,” Proc. DAC, pp. 464-467, 2000. 